\title{
Informes
}

III ENCUENTRO IBEROAMERICANO SOBRE LA CIUDAD; JORNADAS TÉCNICAS SOBRE MEDIO AMBIENTE.

En la sede de Camino de Sta. Teresa del Colegio de Ingenieros Civiles de México tuvo lugar, del 25 de julio al 2 de agosto pasados, el "III Encuentro Iberoamericano sobre la ciudad; jornadas técnicas sobre medio ambiente".

El evento estuvo organizado por el Departamento del Distrito Federal y por Tecniberia, asociación fundada en 1964 para promover la ingeniería, la consultoría y la investigación españolas en el exterior. En la ceremonia inaugural intervinieron el Arq. Juan Gil Elizondo, director general de Reordenación Urbana y Protección Ecológica del DDF, el Dr. Carlos Quesada García, presidente de Tecniberia, y el embajador de España en México, Excmo. Sr. D. Pedro Bermejo Marín, quien transmitió un mensaje del alcalde de Madrid.

A pesar de la presencia de algunos observadores latinoamericanos, el evento tuvo un carácter netamente hispano-mexicano, y se centró en los aspectos técnicos de las políticas ambientales de los gobiernos locales en los grandes asentamientos urbanos, con referencia especial a los casos de Madrid y del D.F., aunque, como se verá, algunas ponencias tuvieron alcances mucho más amplios. La asistencia, bastante nutrida durante los cinco días que duró el Encuentro, estuvo compuesta sobre todo por funcionarios y consultores privados con formación en alguna de las ramas de la ingeniería.

Durante la primera jornada tu vieron lugar Conferencias Plenarias. En los dos días siguientes se desarrollaron los temas de la contaminación del aire, la del agua y la del suelo. El jueves 30 de julio se dedicó a un conjunto de visitas técnicas al Bordo Poniente Xochiaca, a las obras de la Comisión del Lago de Texcoco, y al Vivero Nezahualcóyotl. La última jornada se dedicó al resumen de las relatorías, lectura de conclusiones y clausura a cargo del Lic. Guillermo Cosío Vidaurri. secretario general de Gobierno del DDF.

El contenido de las ponencias fue muy variado. Algunas de ellas se refirieron a la política ambiental en general, incluso desde una perspectiva internacional. Éste fue el caso de la ponencia presentada por la Dirección General del Medio Ambiente de España, leída por el Ing. Antonio Díaz Vargas, y que versaba sobre la incidencia acerca de la política ambiental española del ingreso a la Comunidad Europea.

La mayor parte de las ponencias se refirieron a algunos aspectos técnicos del manejo ambiental en la zona metropolitana de la 
ciudad de México (zмcm) o en la ciudad de Madrid. Podríamos mencionar, en relación con la temática mexicana: "Residuos industriales y hospitalarios" del Arq. René Altamirano; "La contaminación atmosférica por fuentes móviles en la zMCM", de $\mathrm{H}$. Bravo et al. (CCA-UNAM); "Programa de acciones intersectoriales para protección del ambiente en la $\mathrm{ZMCM}$ ", del Lic. Alor Calderón; "Proyecto Texcoco" del Ing. Gerardo Cruikshank; "Los programas de acción del DDF en materia de desarrollo urbano y protección ecológica", del Arq. Francisco de la Vega; "Tratamiento de aguas residuales en el Distrito Federal", del Ing. Francisco Flores; "Desarrollo de tecnologías de bajo costo para el tratamiento de aguas residuales en el D.F.", del M. en I. Pedro A. López; "El manejo social y ambientalmente adecuado del agua en el Valle de México" del Ing. Fernando Ortiz Monasterio; "Control de la contaminación ambiental producida por centrales termoeléctricas", del Ing. Jesús Soria; "La obra pública en zonas de tiraderos de basura: el caso de Sta. Cruz Meyehualco", del Ing. Daniel Valenzuela.

Por el lado español, podrían citarse: "Recolección, tratamiento y aprovechamiento de residuos", del Dr. Gabriel Conde; "Promoción de nuevas tecnologías y tecnologías de bajo costo en el campo de la depuración de las aguas residuales", del Ing. José A. Díaz Lázaro; "Un índice de contaminación atmosférica para áreas urbanas", del Lic. Gonzalo Echagüe; "Técnicas convencionales de depuración de aguas aplicables a grandes urbes e industrias", del Ing. José R. Fernández de Tejada y del Ing. Manuel Fernández del Pino; "Repercusiones de la contaminación atmosférica derivada de la introducción de gas natural en grandes ciudades", del Ing. Santiago Jiménez; "La gestión de los residuos sólidos urbanos" del Ing. Gonzalo Martín Palomo; "Recogida y tratamiento de residuos sólidos urbanos", del Ing. José Murvais; "Tecnologías de bajo costo para el tratamiento de aguas residuales urbanas; la experiencia española", del Quím. José Ma. Puig; "Ubicación e integración paisajística de tiraderos", del Biól. Francisco Suárez.

Un último grupo de ponencias se refiere a una temática más amplia: "La legislación ambiental en México" del Ing. Juan Feo. Bueno; "Estudios de impacto ambiental y reservas de la biosfera", del M. en C. Pedro Reyes; "Plan de Saneamiento de la Cuenca del Río Guadalquivir, Andalucía", del Ing. Román del Río.

Como es habitual en este tipo de Encuentros, la calidad de las ponencias fue bastante desigual. Las menos útiles fueron aquéllas, afortunadamente muy escasas, que asumieron un carácter oficialista en detrimento de un análisis técnico, objetivo, del problema abordado. Varias de ellas no se pudieron reproducir y repartir. 
El evento fue muy positivo en cuanto permitió contactos formales e informales entre técnicos de uno y otro lado del Atlántico, y sentó las bases para una discusión acerca de las diferentes condiciones en las que se verifican los procesos de toma de decisiones tecnológicas. En lo sucesivo se requeriría ampliar y sistematizar las comparaciones entre las diferentes situaciones socio-ambientales, para lograr una mayor convergencia de los conocimientos técnicos acumulados. Se preve dar continuidad a estos Encuentros, el próximo de los cuales se celebrará en Madrid, en fecha a precisar posteriormente.

Fernando Tudela

\section{CONSTITUCIÓN DE LA RED NACIONAL DE FORMACIÓN AMBIENTAL (SEDUE)}

En 1983 se estableció a nivel regional la Red de Formación Ambien: tal para América Latina y El Caribe, con la finalidad de apoyar el trabajo de las instituciones nacionales y subregionales mediante investigación, información y capacitación en materia ambiental, así como asegurar la cooperación técnica entre los países de la región.

El gobierno mexicano, por medio de la Secretaría de Desarrollo Urbano y Ecología (SEDUE) convocó, en marzo de 1985, a las instituciones de educación superior para constituir en México la Red Nacional de Formación Ambiental.

Los objetivos de la Red son: coordinar el trabajo de las instituciones dedicadas a la investigación y a la formación ambiental con los sectores público, social y privado; asesorar a las instituciones responsables del medio ambiente en materia de evaluación, planeación y gestión, y proponer mecanismos viables para promover la investigación, la asesoría y la especialización respecto al manejo de los recursos naturales. La Red busca enfocar de manera multidisciplinaria el tema del medio ambiente.

Pueden ser miembros de la Red: instituciones académicas, de investigación y de docencia, de nivel superior; asociaciones y organizaciones civiles; organismos gubernamentales y organismos internacionales.

La Red funciona con apoyo en un punto focal nacional que tiene la responsabilidad de coordinar horizontalmente a los integrantes; actúa como enlace con la Red Regional de Formación Ambiental para América Latina y El Caribe, y con las redes nacionales de los países integrantes de la región. Coordina, además, las acciones que cada año se incluyen en el Programa de Actividades de la Red.

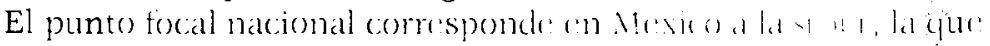


representa al país ante la Oficina Regional para América Latina y El Caribe (ORIPLC:) del Programa de las Naciones Unidas para el Medio Ambiente (PNUMA).

Para llevar a cabo los trabajos de organización de la Red se realizaron cuatro reuniones regionales; en cada una se eligió a un coordinador para fungir como Punto Focal Regional (PFR). Hasta el momento se han establecido las siguientes regiones: Región Centro: Distrito Federal; Región Centro-Este: Guerrero, México, Morelos, Oaxaca, Puebla, Tlaxcala y Veracruz (PFR); Región Centro-Oeste: Colima, Guanajuato, Hidalgo, Jalisco, Michoacán, Querétaro y Aguascalientes (PFR); Región Norte: Coahuila, San Luis Potosí, Durango, Nuevo León, Tamaulipas, Zacatecas, Chihuahua (PFR); Región Noroeste: Baja California, Nayarit, Sinaloa, Sonora, Baja California Sur $\left(P I^{R}\right)$.

Para promover el intercambio y la actualización, la sedue elaboró un Paquete de Comunicación que contiene un directorio de los organismos implicados en la formación ambiental y además propicia relaciones directas entre los interesados.

El Paquete de Comunicación contiene cédulas de identificación realizadas por distintos organismos especializados. Destacan: el Estudio sobre la Incorporación de Formación Ambiental a la Educación Superior de México que realizó El Colegio de México; la Recopilación de Información Científica y Tecnológica en el ámbito del sector Desarrollo Urbano, Vivienda y Ecología. También se utilizaron las recopilaciones hechas por los puntos focales regionales. Además se incluyó información del Directorio de Universidades e Institutos de ANIIEs, la Colección de Inventarios sobre Ingenieria de, la st.P, el Catalogo de Instituciones y Unidades que realizan Investigación $\mathrm{y}$ Desarrollo Experimental del conscit y el documento "Quién es quién en la Ecologia", del Instituto stiu't'.

En el mismo paquete se incluye una relación de las asociaciones civiles interesadas en temas del medio ambiente.

El Paquete de Comunicación tiene como principales objetivos: organizar y sistematizar la información que se produce en el país sobre investigación y formación ambiental; propiciar el contacto entre las instituciones, sus investigadores, los grupos sociales y las dependencias públicas relacionadas con el ambiente; apoyar con información a las instituciones que inicien tareas de formación ambiental. 